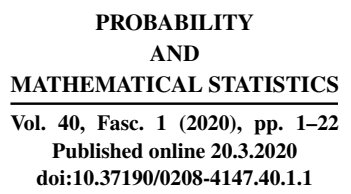

\title{
ON POTENTIAL THEORY OF HYPERBOLIC BROWNIAN MOTION WITH DRIFT
}

BY

\author{
GRZEGORZ SERA F IN* (WROCŁAW)
}

\begin{abstract}
Consider the $\lambda$-Green function and the $\lambda$-Poisson kernel of a Lipschitz domain $U \subset \mathbb{H}^{n}=\left\{x \in \mathbb{R}^{n}: x_{n}>0\right\}$ for hyperbolic Brownian motion with drift. We provide several relationships that facilitate studying those objects and explain somewhat their nature. As an application, we yield uniform estimates for sets of the form $S_{a, b}=\left\{x \in \mathbb{H}^{n}: x_{n}>a\right.$, $\left.x_{1} \in(0, b)\right\}, a, b>0$, which covers and extends existing results of that kind.
\end{abstract}

2020 Mathematics Subject Classification: Primary 60J60; Secondary $58 \mathrm{~J} 65$.

Key words and phrases: hyperbolic space, hyperbolic Brownian motion with drift, $\lambda$-Poisson kernel, $\lambda$-Green function.

\section{INTRODUCTION}

Hyperbolic Brownian motion (HBM) is a canonical diffusion in real hyperbolic space with half the Laplace-Beltrami operator as generator. This process is a natural counterpart of the classical Brownian motion and plays a crucial role in the probabilistic approach to potential theory on hyperbolic space. On the other hand, HBM is closely related to geometric Brownian motion and the Bessel process [2], [25]. It also has some applications to physics [13] and risk theory in financial mathematics [10], [26]. Properties of HBM has been significantly developed in [1], [2], [14], [18] and other papers. The main objects of study, in the context of potential theory on hyperbolic spaces, include the $\lambda$-Green function and the $\lambda$-Poisson kernel of subdomains. They were recently intensively investigated for particular sets (see e.g. [6], [7], [9], [17], [22]). The main result of the paper (Theorem 3.1] implies that studying the aforementioned objects leads to HBM with drift. For this reason, our approach is based on the process with drift from the outset.

* The author was supported by the Faculty of Pure and Applied Mathematics, Wrocław University of Science and Technology.

(c) Probability and Mathematical Statistics, 2020 
We denote by $X^{(\mu)}=\left\{X^{(\mu)}(t)\right\}_{t \geqslant 0}, \mu \in \mathbb{R}$, a HBM with drift on the halfspace model $\mathbb{H}^{n}=\left\{x \in \mathbb{R}^{n}: x_{n}>0\right\}$ of the $n$-dimensional real hyperbolic space. The generator of the process is $\frac{1}{2} \Delta_{\mu}$, where

$$
\Delta_{\mu}=x_{n}^{2} \sum_{k=1}^{n} \frac{\partial^{2}}{\partial x_{k}^{2}}-(2 \mu-1) x_{n} \frac{\partial}{\partial x_{n}} .
$$

Note that $\Delta_{(n-1) / 2}$ is the Laplace-Beltrami operator and therefore $\mu=(n-1) / 2$ corresponds to the standard HBM. In this paper, we focus mostly on $\mu>0$, since the main motivation of studying HBM with drift, mentioned in the first paragraph, concerns positive values of $\mu$. Furthermore, potential theories for opposite indices are associated to each other (see Remark 3.1 after Theorem 3.1), which allows us to study only the positive ones.

Let us denote by $\tau_{U}^{\mu}=\inf \left\{t: X^{(\mu)}(t) \notin U\right\}$ the first exit time of the process from the domain $U$. The objective of the paper is to examine the $\lambda$-Green function $G_{U}^{(\mu), \lambda}(x, y)$ and the $\lambda$-Poisson kernel $P_{U}^{(\mu), \lambda}(x, y)$ of $U$, defined by

$$
\begin{aligned}
& G_{U}^{(\mu), \lambda}(x, y)=\int_{0}^{\infty} e^{-\lambda t} \mathbb{E}^{x}\left[t<\tau_{U}^{\mu} ; X^{(\mu)}(t) \in d y\right] d t / d y, \quad x, y \in U, \\
& P_{U}^{(\mu), \lambda}(x, y)=\mathbb{E}^{x}\left[e^{-\lambda \tau_{U}^{\mu}} ; X^{(\mu)}\left(\tau_{U}^{\mu}\right) \in d y\right] / d y, \quad x \in U, y \in \partial U .
\end{aligned}
$$

In the formula for $P_{U}^{(\mu), \lambda}(x, y)$ we assume additionally that $\tau_{U}^{\mu}<\infty$ a.s. if $\lambda>0$. Furthermore, for $\lambda=0$ the above objects become the Green function and the Poisson kernel, which we denote by $G_{U}^{(\mu), 0}(x, y)=G_{U}^{(\mu)}(x, y)$ and $P_{U}^{(\mu), 0}(x, y)=$ $P_{U}^{(\mu)}(x, y)$, respectively. Those functions are fundamental objects in potential theory on $\mathbb{H}^{n}$. Precisely, they describe solutions for the Dirichlet problem involving the operator $\Delta_{\mu}$. In particular, the Green function appears to be the kernel of the inverse operator to $\Delta_{\mu}$. The $\lambda$-Green function and the $\lambda$-Poisson kernel take over the leading role when the operator $\Delta_{\mu}-\lambda I$ is considered. The $\lambda$-Green function may then be understood as the resolvent kernel for $\Delta_{\mu}$, and the $\lambda$-Poisson kernel recovers $\lambda$-harmonic $\left(\left(\Delta_{\mu}-\lambda I\right)\right.$-harmonic) functions from boundary conditions. The following relationships are provided in Theorem 3.1 .

$$
\begin{aligned}
& G_{U}^{(\mu), \lambda}(x, y)=\left(\frac{x_{n}}{y_{n}}\right)^{\mu-\eta} G_{U}^{(\eta)}(x, y), \\
& P_{U}^{(\mu), \lambda}(x, y)=\left(\frac{x_{n}}{y_{n}}\right)^{\mu-\eta} P_{U}^{(\eta)}(x, y),
\end{aligned}
$$

where $\mu \in \mathbb{R}$ and $\eta=\sqrt{\mu^{2}+2 \lambda}$. The main consequence of this result is that the study of $\lambda$-Green functions and $\lambda$-Poisson kernels can be reduced to the case $\lambda=0$. Furthermore, examining these objects for the standard hyperbolic Brownian motion leads naturally to HBM with drift, which is a substantial motivation to study that process. 
If $\lambda>0$ and $\tau_{U}^{\mu}=\infty$ with positive probability, the $\lambda$-Poisson kernel defined in a classical way becomes degenerate. Indeed, since $\left\{\tau_{U}^{\mu}=\infty\right\}=\left\{X_{n}\left(\tau_{U}^{\mu}\right)=0\right\}$ a.s., the right-hand side of $(1.3)$ vanishes on $\partial U \cap P$, where $P=\left\{x \in \mathbb{R}^{n}\right.$ : $\left.x_{n}=0\right\}$ and $\partial U$ is the boundary of $U$ in Euclidean metric (in $\mathbb{R}^{n}$ ). This effect is due to a specific behavior of $\lambda$-harmonic functions near $P$. The definition (1.3) does not take this behavior into consideration. To discuss this issue more precisely we recall an analytical interpretation of the $\lambda$-Poisson kernel as an integral kernel solving the Dirichlet problem. Then we reformulate the problem and solve it by an integral kernel of the form corresponding to (1.4).

Another important result of the paper is Theorem 3.3 where we show that the Green function and the Poisson kernel for HBM with drift can be easily expressed by analogous objects for Brown-Bessel diffusion. This general method was introduced by Molchanov and Ostrovskii [20] (see also [19]). Finally, in Theorem 3.4 we relate potential theory on $\mathbb{H}^{n}$ to the classical one on the Euclidean space $\mathbb{R}^{2 n}$. However, that result concerns HBM without drift and the relevant set is modified. As an application of general results we provide uniform estimates of the Green function and the Poisson kernel of the set $S_{a, b}=\left\{x \in \mathbb{H}^{n}: x_{n}>a, x_{1} \in(0, b)\right\}$, $a, b>0$. This set may seem very special, but studying it is motivated by geometry: the hyperplanes $x_{n}=a$ are horocycles and the hyperplanes $x_{1}=b$ are geodesics in $\mathbb{H}^{n}$. Moreover, constants in the estimates depend only on the dimension and the parameter $\mu$, and manipulation of parameters $a$ and $b$ lets us recover and improve existing results for sets like $D_{a}=\left\{x \in \mathbb{H}^{n}: x_{n}>a\right\}, H=\left\{x \in \mathbb{H}^{n}: x_{1}>0\right\}$, $S_{b}=\left\{x \in \mathbb{H}^{n}: x_{1} \in(0, b)\right\}$ (see [3], [8], [17], [22]).

The paper is organized as follows. The "Preliminaries" start with a short description of the Bessel process and related objects. The process killed when exiting the half-line $(a, \infty), a>0$, is also considered. Next, the hyperbolic space $\mathbb{H}^{n}$ and HBM with drift are introduced. In Section 3 we collect several relationships which simplify the study of the $\lambda$-Green function and the $\lambda$-Poisson kernel of subdomains of $\mathbb{H}^{n}$. Section 4 is devoted to estimates of the Green function and the Poisson kernel of the set $S_{a, b}$. In the Appendix, one can find an integral lemma which is intensively exploited in Section 4.

\section{PRELIMINARIES}

2.1. Notation. We denote by $|\cdot|$ the Euclidean norm $|x|=\sqrt{x_{1}^{2}+\cdots+x_{n}^{2}}$ of a given vector $x \in \mathbb{R}^{n}$. The estimates below use the following notation: for two positive functions $f, g: X \rightarrow(0, \infty)$ we write $f \approx g$ if there exists a constant $c>1$ such that $1 / c \leqslant f / g \leqslant c$ for every $x \in X$. If the constant $c$ depends on an additional parameter, we write this parameter over $\approx$.

2.2. Bessel process. We denote by $R^{(\nu)}=\left\{R^{(\nu)}(t)\right\}_{t \geqslant 0}$ the Bessel process with index $\nu<0$ starting from $R^{(\nu)}(0)=x>0$. Nonnegative indices are also considered in the literature, but they are irrelevant from our point of view. For $\nu \leqslant-1$ 
the point 0 is killing and the process hits it a.s. In the case $-1<\nu<0$ we impose the killing condition at 0 . The transition density function of the process is given by (see [5, p. 134])

$$
g^{(\nu)}(t, x, y)=\frac{y}{t}\left(\frac{y}{x}\right)^{\nu} \exp \left(-\frac{x^{2}+y^{2}}{2 t}\right) I_{|\nu|}\left(\frac{x y}{t}\right), \quad \nu<0, x, y>0,
$$

where $I_{\nu}(z)$ is the modified Bessel function of the first kind.

Let $B=\{B(t)\}_{t \geqslant 0}$ be the one-dimensional Brownian motion starting from zero. The Bessel process is related to the geometric Brownian motion $\{x \exp (B(t)+\nu t)\}_{t \geqslant 0}, x>0$, by the Lamperti relation

$$
\{x \exp (B(t)+\nu t)\}_{t \geqslant 0} \stackrel{d}{=}\left\{R^{(\nu)}\left(A_{x}^{(\nu)}(t)\right)\right\}_{t \geqslant 0},
$$

where the integral functional $A_{x}^{(\nu)}(t)$ is defined by

$$
A_{x}^{(\nu)}(t)=x^{2} \int_{0}^{t} \exp (2 B(s)+2 \nu s) d s .
$$

The density function $f_{x, t}^{(\nu)}(u, v)$ of a vector $\left(A_{x}^{(\nu)}(t), x \exp (B(t)+\nu t)\right)$ was computed in [26] and is given by

$$
f_{x, t}^{(\nu)}(u, v)=\left(\frac{v}{x}\right)^{\nu} e^{-\nu^{2} t / 2} \frac{1}{u v} \exp \left(-\frac{x^{2}+v^{2}}{2 u}\right) \theta_{x v / u}(t), \quad x, u, v, t>0 .
$$

Here, the function $\theta_{r}(t)$ satisfies (see [24])

$$
\int_{0}^{\infty} e^{-\lambda t} \theta_{r}(t) d t=I_{\sqrt{2 \lambda}}(r)
$$

Note that the function $f_{x, t}^{(\nu)}(u, v)$ is also closely related to the Hartman-Watson law (see [15]). Furthermore, (2.4), (2.5) and (2.1) imply

$$
\begin{aligned}
\int_{0}^{\infty} f_{x, t}^{(\nu)}(u, v) d t & =\left(\frac{v}{x}\right)^{\nu} \frac{1}{u v} \exp \left(-\frac{x^{2}+v^{2}}{2 u}\right) I_{|\nu|}\left(\frac{x v}{u}\right) \\
& =\frac{1}{v^{2}} g^{(\nu)}(u, x, v) .
\end{aligned}
$$

The Bessel process with a negative index $\nu$ and starting from $x>a, a>0$, leaves the half-line $(a, \infty)$ with probability 1 . The transition density function of the process killed on exiting $(a, \infty)$ has been estimated in [4]:

$$
\begin{aligned}
& g_{a}^{(\nu)}(t ; x, y) \\
& \quad \approx \frac{(x-a)(y-a)}{t+(x-a)(y-a)}\left(\frac{x^{2}}{t+x y}\right)^{|\nu|-1 / 2} \frac{1}{\sqrt{t}} \exp \left(-\frac{(x-y)^{2}}{2 t}\right),
\end{aligned}
$$

where $x, y>a, t>0$. Furthermore, let us denote by $q_{a}^{(\nu)}(t ; x), a>0$, the density 
function of the first hitting time of the point $a$ by the Bessel process. It has been estimated in [8]:

$$
\begin{aligned}
& q_{a}^{(\nu)}(t ; x) \approx \frac{x-a}{t^{3 / 2}} \frac{x^{2|\nu|-1}}{(t+a x)^{|\nu|-1 / 2}} \exp \left(-\frac{(x-y)^{2}}{2 t}\right), \\
& t>0, x>a, \nu<0 \text {. }
\end{aligned}
$$

In fact, the above formula corrects a minor mistake of [8]. Precisely, Theorems 4 and 8 in [8] providing estimates when $x>t$ and when $x<t$, respectively, are correct, but the formula (15) combining them is wrong: $t^{|\mu|-1 / 2}+x^{|\mu|-1 / 2}$ in the denominator should read $(t+x)^{|\mu|-1 / 2}$.

2.3. Hyperbolic space and hyperbolic Brownian motion with drift. We consider the half-space model of the real hyperbolic space

$$
\mathbb{H}^{n}=\left\{x \in \mathbb{R}^{n}: x_{n}>0\right\}, \quad n=1,2,3, \ldots
$$

The formula for hyperbolic distance is given by

$$
\cosh d_{\mathbb{H}^{n}}(x, y)=\left(1+\frac{|x-y|^{2}}{2 x_{n} y_{n}}\right), \quad x, y \in \mathbb{H}^{n} .
$$

The unique, up to a constant factor, second order elliptic differential operator on $\mathbb{H}^{n}$, annihilating constant functions, which is invariant under isometries of the space, is the Laplace-Beltrami operator $\Delta_{(n-1) / 2}($ cf. $[1.1)$ ).

Hyperbolic Brownian motion with drift is a process $X^{(\mu)}=\left\{X^{(\mu)}(t)\right\}_{t \geqslant 0}$ starting from $X^{(\mu)}(0)=x \in \mathbb{H}^{n}$ whose generator is $\frac{1}{2} \Delta_{\mu}$. The parameter $\mu$ is called an index and the drift is equal to $\mu-(n-1) / 2$. Note that for $\mu=(n-1) / 2$ we obtain the standard HBM (without drift).

Let us denote by $B(t)=\left(B_{1}(t), \ldots, B_{n}(t)\right)$ the classical Brownian motion in $\mathbb{R}^{n}$ starting from $\left(x_{1}, \ldots, x_{n-1}, 0\right)$. Then HBM with drift may be represented in terms of $B(t)$ as follows (see [3]):

$$
X^{(\mu)}(t) \stackrel{d}{=}\left(B_{1}\left(A_{x_{n}}^{(-\mu)}(t)\right), \ldots, B_{n-1}\left(A_{x_{n}}^{(-\mu)}(t)\right), x_{n} \exp \left(B_{n}(t)-\mu t\right)\right) .
$$

Here, the integral functional $A_{x_{n}}^{(-\mu)}(t)$, defined by 2.3$)$, is associated with $B_{n}(t)$. In addition, using the Lamperti relation, we get

$$
\left\{X^{(\mu)}(t) ; t \geqslant 0\right\} \stackrel{(d)}{=}\left\{Y\left(A_{x_{n}}^{(-\mu)}(t)\right) ; t \geqslant 0\right\},
$$

where

$$
Y(t)=\left(B_{1}(t), \ldots, B_{n-1}(t), R^{(-\mu)}(t)\right),
$$

and $R^{(-\mu)}(t)$ is the Bessel process with index $-\mu$ starting from $x_{n}$ and independent of $\left(B_{1}(t), \ldots, B_{n-1}(t)\right)$. 


\section{GENERAL RESULTS}

3.1. Reduction to $\lambda=0$. In this subsection we provide precise relationships which connect the $\lambda$-Green function and the $\lambda$-Poisson kernel with analogous objects for $\lambda=0$ and for the process with a different drift. This lets us reduce $\lambda$-potential theory to the case $\lambda=0$. The only cost we pay is the above-mentioned change of the drift of the process.

THEOREM 3.1. Let $\mu \in \mathbb{R}, U$ a domain in $\mathbb{H}^{n}$ and $\lambda \geqslant 0$. Then

$$
G_{U}^{(\mu), \lambda}(x, y)=\left(\frac{x_{n}}{y_{n}}\right)^{\mu-\eta} G_{U}^{(\eta)}(x, y), \quad x, y \in U,
$$

where $\eta=\sqrt{\mu^{2}+2 \lambda}$. If additionally $\tau_{U}^{\mu}, \tau_{U}^{\eta}<\infty$ a.s., then

$$
P_{U}^{(\mu), \lambda}(x, y)=\left(\frac{x_{n}}{y_{n}}\right)^{\mu-\eta} P_{U}^{(\eta)}(x, y), \quad x \in U, y \in \partial U .
$$

Proof. The last coordinate of the process $X^{(\mu)}(t)$ can be expressed in the form $X_{n}^{(\mu)}(t)=x_{n} \exp \left(W^{(\eta-\mu)}(t)-\eta t\right)$, where $W^{(\eta-\mu)}(t)=B_{n}(t)+(\eta-\mu) t$ and $B_{n}$ is a one-dimensional Brownian motion. By the Girsanov theorem, the process $\left\{W^{(\eta-\mu)}(t)\right\}_{0 \leqslant t \leqslant T}$ is, for every $T>0$, a standard Brownian motion with respect to the measure $Q_{T}$ given by

$$
\frac{d Q_{T}}{d \mathbb{P}}=\exp \left((\mu-\eta) B_{n}(T)-\frac{1}{2}(\eta-\mu)^{2} T\right)=M(T) .
$$

This implies that the process $\left\{X^{(\mu)}(t)\right\}_{0 \leqslant t \leqslant T}$ considered with respect to the measure $Q_{T}$ is a hyperbolic Brownian motion with drift with index $\eta$. Hence, for every $t \leqslant T$ and every Borel set $A \subset U$ we get

$$
\mathbb{E}^{x}\left[t<\tau_{U}^{\eta} ; X^{(\eta)}(t) \in A\right]=\mathbb{E}^{x}\left[t<\tau_{U}^{\mu} ; M(T) ; X^{(\mu)}(t) \in A\right] .
$$

Observe that $M(t)$ is an $\mathcal{F}_{t}$-martingale and it may be rewritten as

$$
M(t)=\left[\exp \left(B_{n}(t)-\mu t\right)\right]^{\mu-\eta} e^{-\left(\eta^{2}-\mu^{2}\right) t / 2}=x_{n}^{\eta-\mu} e^{-\lambda t}\left[X_{n}^{(\mu)}(t)\right]^{\mu-\eta} .
$$

Furthermore, denote by $\mathcal{F}_{t}$ the $\sigma$-field generated by $\left\{X_{s}^{(\mu)}\right\}_{0 \leqslant s \leqslant t}$. The set $\left\{t<\tau_{U}^{\mu}\right\}$ is then $\mathcal{F}_{t}$-measurable and for $t \leqslant T$ we get

$$
\begin{aligned}
\mathbb{E}^{x}\left[t<\tau_{U}^{\eta} ; X^{(\eta)}(t) \in A\right] & =\mathbb{E}^{x}\left[\mathbb{E}^{x}\left[t<\tau_{U}^{\mu} ; M(T) ; X^{(\mu)}(t) \in A \mid \mathcal{F}_{t}\right]\right] \\
& =\mathbb{E}^{x}\left[t<\tau_{U}^{\mu} ; \mathbb{E}^{x}\left[M(T) \mid \mathcal{F}_{t}\right] ; X^{(\mu)}(t) \in A\right] \\
& =\mathbb{E}^{x}\left[t<\tau_{U}^{\mu} ; M(t) ; X^{(\mu)}(t) \in A\right] \\
& =x_{n}^{\eta-\mu} e^{-\lambda t} \mathbb{E}^{x}\left[t<\tau_{U}^{\mu} ;\left(X_{n}^{(\mu)}(t)\right)^{\mu-\eta} ; X^{(\mu)}(t) \in A\right] .
\end{aligned}
$$


Since there is no upper bound of $T$, the above equalities hold for every $t \geqslant 0$ and consequently

$$
\begin{aligned}
\int_{A} G_{U}^{(\eta)}(x, y) d y & =\int_{0}^{\infty} \mathbb{E}^{x}\left[t<\tau_{U}^{\eta} ; X^{(\eta)}(t) \in A\right] d t \\
& =x_{n}^{\eta-\mu} \int_{0}^{\infty} e^{-\lambda t} \mathbb{E}^{x}\left[t<\tau_{U}^{\mu} ;\left(X_{n}^{(\mu)}(t)\right)^{\mu-\eta} ; X^{(\mu)}(t) \in A\right] d t \\
& =x_{n}^{\eta-\mu} \int_{A} y_{n}^{\mu-\eta} G_{U}^{(\mu), \lambda}(x, y) d y
\end{aligned}
$$

which proves the formula (3.1). Let us now prove the latter assertion of the theorem. As before, for every Borel $C \subset \partial U$ we get

$$
\begin{aligned}
\mathbb{E}^{x}\left[\tau_{U}^{\eta}<T ; X^{(\eta)}\left(\tau_{U}^{\eta}\right) \in C\right] & =\mathbb{E}^{x}\left[\tau_{U}^{\mu}<T ; M(T) ; X^{(\mu)}\left(\tau_{U}^{\mu}\right) \in C\right] \\
& =\mathbb{E}^{x}\left[\tau_{U}^{\mu}<T ; \mathbb{E}^{x}\left[M(T) \mid \mathcal{F}_{\tau_{U}^{\mu}}\right] ; X^{(\mu)}\left(\tau_{U}^{\mu}\right) \in C\right],
\end{aligned}
$$

where

$$
\mathcal{F}_{\tau_{U}^{\mu}}=\left\{A \in \mathcal{F}_{\infty}:(\forall t \geqslant 0) A \cap\left\{\tau_{U}^{\mu}<t\right\} \in \mathcal{F}_{t}\right\}
$$

Using Doob's optional stopping theorem we obtain

$$
\begin{aligned}
& \mathbb{E}^{x}\left[\tau_{U}^{\eta}<T ; X^{(\eta)}\left(\tau_{U}^{\eta}\right) \in C\right]=\mathbb{E}^{x}\left[\tau_{U}^{\mu}<T ; M\left(\tau_{U}^{\mu}\right) ; X^{(\mu)}\left(\tau_{U}^{\mu}\right) \in C\right] \\
& =x_{n}^{\eta-\mu} \mathbb{E}^{x}\left[\tau_{U}^{\mu}<T ;\left(X_{n}^{(\mu)}\left(\tau_{U}^{\mu}\right)\right)^{\mu-\eta} e^{-\lambda \tau_{U}^{\mu}} ; X^{(\mu)}\left(\tau_{U}^{\mu}\right) \in C\right] .
\end{aligned}
$$

The next step is to take the limit as $T \rightarrow \infty$. By the assumption $\tau_{U}^{\mu}, \tau_{U}^{\eta}<\infty$ a.s., the monotone convergence theorem gives

$$
\mathbb{E}^{x}\left[X^{(\eta)}\left(\tau_{U}^{\eta}\right) \in C\right]=x_{n}^{\eta-\mu} \mathbb{E}^{x}\left[\left(X_{n}^{(\mu)}\left(\tau_{U}^{\mu}\right)\right)^{\mu-\eta} e^{-\lambda \tau_{U}^{\mu}} ; X^{(\mu)}\left(\tau_{U}^{\mu}\right) \in C\right],
$$

which is equivalent to 3.2 .

REMARK 3.1. Another significance of the above theorem is that it reveals some kind of duality between potential theories for opposite values of $\mu$. Taking $\lambda=0$ and $-\mu$ instead of $\mu$ we get, for $\mu>0$,

$$
\begin{aligned}
& G_{U}^{(-\mu)}(x, y)=\left(\frac{x_{n}}{y_{n}}\right)^{-2 \mu} G_{U}^{(\mu)}(x, y), \\
& P_{U}^{(-\mu)}(x, y)=\left(\frac{x_{n}}{y_{n}}\right)^{-2 \mu} P_{U}^{(\eta)}(x, y) .
\end{aligned}
$$

We turn now to the case when $\mathbb{P}^{x}\left(\tau_{U}^{\mu}=\infty\right)>0$. Since $X_{n}^{(\mu)}(\infty)=0$ (cf. (2.10), the right-hand side of (1.3) vanishes at $y_{n}=0$ for $\lambda>0$. This situation is singular, especially from the analytical point of view. Namely, the $\lambda$-Poisson kernel 
is supposed to solve the Dirichlet problem with a given boundary condition. In our situation, the condition on the set $P_{U}^{(\mu), \lambda}(x, y)=0\left(=\partial U \cap\left\{y_{n}=0\right\}\right)$ does not affect the behaviour of the solution in the neighbourhood of that set. However, some examples show that this behaviour is relevant and setting boundary conditions only on the set $\partial U \cap\left\{y_{n}>0\right\}$ results in an infinite number of solutions. Furthermore, we can observe that multiplying the right-hand side of 3.2 by $y_{n}^{\mu-\eta}$ and enlarging the set $U$ we obtain a nontrivial object. Finally, if a function $f(x)$ is $\lambda$-harmonic for the operator $\frac{1}{2} \Delta_{\mu}$ (i.e. $\frac{1}{2} \Delta_{\mu} f(x)=\lambda f(x)$ ), then the function $x_{n}^{\eta-\mu} f(\mu)$, where $\eta=\sqrt{2 \lambda+\mu^{2}}$, is harmonic for the operator $\frac{1}{2} \Delta_{\eta}$, which comes from

$$
\begin{aligned}
& \frac{1}{2} \Delta_{\eta}\left(x_{n}^{\eta-\mu} f(x)\right) \\
& \quad=x_{n}^{\eta-\mu} \frac{1}{2} \Delta_{\eta} f(x)+(\eta-\mu) x_{n}^{\mu-\eta+1} \frac{\partial f}{\partial x_{n}}(x)-\frac{\eta^{2}-\mu^{2}}{2} x_{n}^{\mu-\eta} f(x) \\
& \quad=x_{n}^{\mu-\eta} \frac{1}{2} \Delta_{\mu} f(x)-\lambda x_{n}^{\mu-\eta} f(x)=0 .
\end{aligned}
$$

One can show (using e.g. [23, Theorem 4]) that every continuous bounded function on a Lipschitz domain $U$, which is harmonic for $\Delta_{\eta}, \eta>0$, has a limit at the boundary of $U$. All this leads us to the following modified Dirichlet problem:

Let $U \subset \mathbb{H}^{n}$ be a Lipschitz domain, $f \in \mathcal{C}_{b}(\partial U)$ and $\lambda>0$. Find a function $u \in \mathcal{C}^{2}(U)$ satisfying the differential equation

$$
\left(\frac{1}{2} \Delta_{\mu} u\right)(x)=\lambda u(x), \quad x \in U
$$

such that the function $x_{n}^{\sqrt{2 \lambda+\mu^{2}}-\mu} u(x)$ is bounded and

$$
\lim _{\substack{x \rightarrow z \\ x \in U}} x_{n}^{\sqrt{2 \lambda+\mu^{2}}-\mu} u(x)=f(z), \quad z \in \partial U .
$$

THEOREM 3.2. The function u satisfying (3.6) and (3.7) is unique and given by

$$
u(x)=x_{n}^{\mu-\eta} \int_{\partial U} f(y) P_{U}^{(\eta)}(x, y) d y,
$$

where $\eta=\sqrt{\mu^{2}+2 \lambda}$.

REMARK 3.2. According to this theorem, we can treat the function $x_{n}^{\mu-\eta} P_{U}^{(\eta)}$ as a kind of $\lambda$-Poisson kernel. We do not recover the formula for the $\lambda$-Poisson kernel from Theorem 3.1, but the only difference is the factor $y_{n}^{\mu-\eta}$.

Proof of Theorem 3.2. Define $h(x)=x_{n}^{\eta-\mu} u(x)=\mathbb{E}^{x}\left[f\left(X^{(\eta)}\left(\tau_{U}^{\eta}\right)\right)\right]$. It is bounded by $\|f\|_{\infty}$ and, by the stochastic continuity of the process $X^{(\mu)}$, satisfies 
condition 3.7). Since $P_{U}^{(\eta)}(x, y)$ is the standard Poisson kernel for the process $X^{(\eta)}(t)$, we have $\Delta_{\eta} h(x)=0$. Thus, similarly to 3.5 , we get $\frac{1}{2} \Delta_{\mu} u(x)=\lambda u(x)$.

To prove the uniqueness of the solution let us consider a sequence of bounded (in hyperbolic metric) sets such that $U_{m} \nearrow U$. For every $m$ the function $u_{\uparrow_{m}}$ satisfies (3.6) and 3.7) for $U_{m}$ in place of $U$ and for $f=u_{\uparrow \partial U_{m}} \in \mathcal{C}_{b}\left(\partial U_{m}\right)$ and it is the unique function with this property (see [12]). Moreover (see [16, Prop. 7.2, p. 364])

$$
u_{\uparrow U_{m}}(x)=\mathbb{E}^{x}\left[e^{-\lambda \tau_{U_{m}}^{\mu}} u\left(X^{(\mu)}\left(\tau_{U_{m}}^{\mu}\right)\right)\right] .
$$

Hence, by Theorem 3.1, we get

$$
u(x)=\mathbb{E}^{x}\left[e^{-\lambda \tau_{U_{m}}^{\mu}} u\left(X^{(\mu)}\left(\tau_{U_{m}}^{\mu}\right)\right)\right]=x_{n}^{\mu-\eta} \mathbb{E}^{x}\left[\left(X^{(\eta)}\left(\tau_{U_{m}}^{\eta}\right)\right)^{\eta-\mu} u\left(X^{(\eta)}\left(\tau_{U_{m}}^{\eta}\right)\right)\right] .
$$

Letting $m \rightarrow \infty$, by the Lebesgue dominated convergence theorem we obtain

$$
u(x)=x_{n}^{\mu-\eta} \mathbb{E}^{x}\left[f\left(X^{(\eta)}\left(\tau_{U}^{\eta}\right)\right)\right]
$$

3.2. Representations involving other processes. Let us define the Green function $G_{U}^{Y}(x, y)$ and the Poisson kernel $P_{U}^{Y}(x, y)$ of the set $U \subset \mathbb{H}^{n}$ for the BrownBessel diffusion $Y(t)$ (see (2.12) ) analogously to the case of HBM with drift, i.e.

$$
\begin{aligned}
& P_{U}^{Y}(x, y):=\mathbb{P}^{x}\left(Y\left(\tau_{U}^{Y}\right) \in d y\right), \quad x \in U, y \in \partial U \\
& G_{U}^{Y}(x, y):=\int_{0}^{\infty} \mathbb{E}^{x}\left[t<\tau_{U}^{Y} ; Y(t) \in d y\right] d t, \quad x, y \in U .
\end{aligned}
$$

The next lemma lets us study these objects instead of their counterparts for HBM with drift. The main advantage of this result comes from coordinate independence of the process $Y(t)$ and from the fact that this process is relatively well known.

THEOREM 3.3. For any domain $U \subset \mathbb{H}^{n}$ we have

$$
\text { (i) } P_{U}^{(\mu)}(x, y)=P_{U}^{Y}(x, y), \quad \text { (ii) } G_{U}^{(\mu)}(x, y)=\frac{1}{y_{n}^{2}} G_{U}^{Y}(x, y) \text {. }
$$

REMARK 3.3. The first assertion may be found in [17]; however, the proof is short so we repeat it for convenience of the reader. The other assertion is proved for the set $D_{a}=\left\{x \in \mathbb{H}^{n}: x_{n}>a\right\}$ in [3], but the proof given below is much simpler and covers general sets.

Proof of Theorem 3.3. According to 2.11, the process $Z(t)=Y\left(A_{x_{n}}^{(-\mu)}(t)\right)$ is a HBM with drift. Since the functional $A_{x_{n}}^{(-\mu)}(t)$ is continuous and increasing a.s., we have $\tau_{U}^{Y}=A_{x_{n}}^{(-\mu)}\left(\tau_{U}^{\mu}\right)$ a.s. Thus

$$
X^{(\mu)}\left(\tau_{U}^{\mu}\right) \stackrel{d}{=} Z\left(\tau_{U}^{\mu}\right)=Y\left(A_{x_{n}}^{(-\mu)}\left(\tau_{U}^{\mu}\right)\right) \stackrel{\text { a.s. }}{=} Y\left(\tau_{U}^{Y}\right) .
$$


Denote by $p^{(\mu)}(t ; x, y)$ the transition density function (with respect to the Lebesgue measure) of the process $Z(t)$. By the Hunt formula and the Fubini-Tonelli theorem we have

$$
\begin{aligned}
\int_{0}^{\infty} \mathbb{E}^{x}[t< & \left.\tau_{U}^{\mu} ; Z(t) \in d y\right] d t \\
& =\int_{0}^{\infty} p^{(\mu)}(t ; x, y)-\mathbb{E}^{x}\left[t>\tau_{U}^{\mu} ; p^{(\mu)}\left(t-\tau_{U}^{\mu}, Z\left(\tau_{U}^{Z}\right), y\right)\right] d t \\
& =\int_{0}^{\infty} p^{(\mu)}(t ; x, y) d t-\mathbb{E}^{x}\left[\int_{\tau_{U}^{Z}}^{\infty} p^{(\mu)}\left(t-\tau_{U}^{Z}, Z\left(\tau_{U}^{\mu}\right), y\right) d t\right] \\
& =\int_{0}^{\infty} p^{(\mu)}(t ; x, y) d t-\mathbb{E}^{x}\left[\int_{0}^{\infty} p^{(\mu)}\left(t, Z\left(\tau_{U}^{\mu}\right), y\right) d t\right] .
\end{aligned}
$$

Using representation (2.10) and formulae (2.4), 2.6) we get

$$
\begin{aligned}
\int_{0}^{\infty} p^{(\mu)}(t ; x, y) d t & =\int_{0}^{\infty} \int_{0}^{\infty} \frac{1}{(2 \pi u)^{(n-1) / 2}} e^{-(\tilde{x}-\tilde{y})^{2} /(2 u)} f_{x_{n}, t}^{(\mu)}\left(u, y_{n}\right) d u d t \\
& =\frac{1}{y_{n}^{2}} \int_{0}^{\infty} \frac{1}{(2 \pi u)^{(n-1) / 2}} e^{-(\tilde{x}-\tilde{y})^{2} /(2 u)} g^{(-\mu)}\left(u ; x_{n}, y_{n}\right) d u
\end{aligned}
$$

where $\tilde{x}=\left(x_{1}, \ldots, x_{n-1}\right) \in \mathbb{R}^{n-1}$ and $g^{(\nu)}(u ; x, y)$ is the transition density function of the Bessel process with index $\nu$ starting from $x$. We identify the last integrand as the transition density function of the process $Y(t)$. Since $Z\left(\tau_{U}^{\mu}\right) \stackrel{d}{=} Y\left(\tau_{U}^{Y}\right)$ and the property 3.8 can be derived also for the process $Y(t)$, we obtain (ii).

The above theorem, together with the scaling properties of the standard Brownian motion and the Bessel process, gives us the following scaling properties of the Green function and the Poisson kernel for HBM with drift.

Corollary 3.1. For any domain $U \subset \mathbb{H}^{n}$ and $a>0$ we have

$$
\begin{aligned}
& G_{a U}^{(\mu)}(x, y)=\frac{1}{a^{n}} G_{U}^{(\mu)}\left(\frac{x}{a}, \frac{y}{a}\right), \\
& P_{a U}^{(\mu)}(x, y)=\frac{1}{a^{n-1}} P_{U}^{(\mu)}\left(\frac{x}{a}, \frac{y}{a}\right) .
\end{aligned}
$$

The last theorem in this section shows that the Green function and the Poisson kernel of a set $U$ for HBM (without drift) in $\mathbb{H}^{n}$ may be derived from their counterparts for a somewhat modified set and for the classical Brownian motion in $\mathbb{R}^{2 n}$. This shows that studying potential theory for HBM may be reduced to the classical potential theory. Note that such results for a class of tube domains were obtained in [22]. 
For $A \subset \mathbb{H}^{n}$ we define

$$
A^{+}:=\left\{x \in \mathbb{R}^{2 n}:\left(x_{1}, x_{2}, \ldots, x_{n-1},\left|\left(x_{n}, x_{n+1}, \ldots, x_{2 n}\right)\right|\right) \in A\right\} \subset \mathbb{R}^{2 n},
$$

and for $x \in \mathbb{R}^{n}$ we put

$$
x^{+}=(x, \underbrace{0, \ldots, 0}_{n \text { zeros }}) \in \mathbb{R}^{2 n} .
$$

The general form of the set $A^{+}$may be slightly discouraging, but in many cases it is not very complicated, e.g. for the set $\left\{x \in \mathbb{H}^{n}: x_{n}<a\right\}, a>0$, or for tube domains. Furthermore, we denote by $G_{U}^{B}(x, y)$ and $P_{U}^{B}(x, y)$ the Green function and the Poisson kernel, respectively, of the set $U \subset \mathbb{R}^{2 n}$ for the classical Brownian motion in $\mathbb{R}^{2 n}$.

THEOREM 3.4. For any open set $A \subset \mathbb{H}^{n}$ we have

$$
G_{A}(x, y)=\frac{x_{n}^{n-1}}{y_{n}^{n+1}} \int_{y_{n} S^{n}} G_{A^{+}}^{B}\left(x^{+},(\tilde{y}, w)\right) d \sigma(w), \quad x, y \in A
$$

where $\tilde{y}=\left(y_{1}, \ldots, y_{n-1}\right)$ and $\sigma$ denotes the $n$-dimensional spherical measure on the sphere $y_{n} S^{n}=\left\{z \in \mathbb{R}^{n+1}:|z|=y_{n}\right\}$. Furthermore, if $\partial A \cap\left(\mathbb{R}^{n-1} \times\{0\}\right)$ $=\emptyset$, then

$$
P_{A}(x, y)=\left(\frac{x_{n}}{y_{n}}\right)^{n-1} \int_{y_{n} S^{n}} P_{A^{+}}^{B}\left(x^{+},(\tilde{y}, w)\right) d \sigma(w), \quad x \in A, y \in \partial A .
$$

Proof. By (3.4) and Theorem 3.3 we get

$$
\begin{aligned}
& G_{U}^{\left(-\frac{n-1}{2}\right)}(x, y)=\left(\frac{x_{n}}{y_{n}}\right)^{n-1} G_{U}^{\left(\frac{n-1}{2}\right)}(x, y)=\frac{x_{n}^{n-1}}{y_{n}^{n+1}} G_{U}^{Y}(x, y), \\
& P_{U}^{\left(-\frac{n-1}{2}\right)}(x, y)=\left(\frac{x_{n}}{y_{n}}\right)^{n-1} P_{U}^{\left(\frac{n-1}{2}\right)}(x, y)=\left(\frac{x_{n}}{y_{n}}\right)^{n-1} P_{U}^{Y}(x, y),
\end{aligned}
$$

where $Y(t)=\left(B_{1}(t), \ldots, B_{n-1}(t), R^{\left(\frac{n-1}{2}\right)}(t)\right)$. Since the Bessel process $R^{\left(\frac{n-1}{2}\right)}$ may be interpreted as a norm of an $n+1$-dimensional standard Brownian motion, we obtain

$$
Y \stackrel{d}{=}\left(B_{1}, B_{2}, \ldots, B_{n-1},\left|\left(B_{n}, \ldots, B_{2 n}\right)\right|\right)
$$

where $B=\left(B_{1}, \ldots, B_{2 n}\right)$ is a $2 n$-dimensional Brownian motion starting from $x^{+}$. Let $f$ be a positive function on $\mathbb{H}^{n}$. Following the convention that $G_{A}(x, \cdot)$ 
vanishes on $A^{c}$ we get

$$
\begin{aligned}
& \int_{A} G_{A}^{Y}(x, y) f(y) d y=\int_{0}^{\infty} \mathbb{E}^{x}\left[f(Y) ; t<\tau_{A}^{Y}\right] d t \\
& =\int_{0}^{\infty} \mathbb{E}^{x^{+}}\left[f\left(B_{1}(t), \ldots, B_{n-1}(t),\left|\left(B_{n}(t), \ldots, B_{2 n}(t)\right)\right|\right) ; t<\tau_{A^{+}}^{B}\right] d t \\
& =\int_{\mathbb{R}^{2 n}} f\left(y_{1}, \ldots, y_{n-1}, \sqrt{z_{1}^{2}+\cdots+z_{n+1}^{2}}\right) \\
& \quad \times G_{A^{+}}^{B}\left(x^{+},\left(y_{1}, \ldots, y_{n-1}, w\right)\right) d y_{1} \ldots d y_{n-1} d z \\
& =\int_{\mathbb{H}^{n}} f\left(y_{1}, \ldots, y_{n-1}, y_{n}\right) \int_{y_{n} S^{n}} G_{A^{+}}^{B}\left(x^{+},(\tilde{y}, w)\right) d \sigma(w) d y_{1} \ldots d y_{n} .
\end{aligned}
$$

Furthermore, let $\tau_{A^{+}}^{B}$ be the first exit time of the Brownian motion $B(t)$ from the set $A^{+}$. Then for every positive function $g$ on $\partial A$ we have

$$
\begin{aligned}
\mathbb{E}^{x}\left[g\left(Y\left(\tau_{A}^{Y}\right)\right)\right] \\
\quad=\mathbb{E}^{x^{+}}\left[g\left(B_{1}\left(\tau_{A^{+}}^{B}\right), \ldots, B_{n-1}\left(\tau_{A^{+}}^{B}\right),\left|\left(B_{n}\left(\tau_{A^{+}}^{B}\right), \ldots, B_{2 n}\left(\tau_{A^{+}}^{B}\right)\right)\right|\right)\right] \\
\quad=\int_{\partial A^{+}} g\left(y_{1}, \ldots, y_{n-1}, z_{1}, \ldots, z_{n+1}\right) P_{A^{+}}^{B}\left(x^{+},(\tilde{y}, z)\right) d y_{1} \ldots d y_{n-1} d z \\
\quad=\int_{\partial A} g\left(y_{1}, \ldots, y_{n-1}, y_{n}\right) \int_{y_{n} S^{n}} P_{A^{+}}^{B}\left(x^{+},(\tilde{y}, w)\right) d \sigma(w) d y .
\end{aligned}
$$

Here, $d y$ stands for the induced Lebesgue measure on $\partial A$.

\section{ESTIMATES}

For $a, b>0$ we define

$$
S_{a, b}=\left\{x \in \mathbb{H}^{n}: x_{n}>a, x_{1} \in(0, b)\right\} .
$$

Studying this kind of sets is motivated by hyperbolic geometry. The set $S_{a, b}$ is bounded by three hyperplanes: $P_{1}=\left\{x \in \mathbb{H}^{n}: x_{1}=0\right\}, P_{2}=\left\{x \in \mathbb{H}^{n}\right.$ : $\left.x_{1}=b\right\}$ and $P_{3}=\left\{x \in \mathbb{H}^{n}: x_{n}=a\right\}$. Symmetries with respect to the hyperplanes $P_{1}$ and $P_{2}$ are isometries in $\mathbb{H}^{n}$; the set $P_{3}$ is a horocycle. In this section we estimate the Green function and the Poisson kernel of $S_{a, b}$ uniformly with respect to space variables as well as to the parameters $a$ and $b$. This leads to estimates for some other sets that may be obtained from $S_{a, b}$ by manipulating the values of the parameters.

By $\delta_{u}(w)=w \wedge(u-w), u>0, w \in(0, u)$, we denote the Euclidean distance between $w$ and a complement of the interval $(0, u)$. We clearly have $\delta_{u}(w) \approx$ $w(u-w) / u$. Moreover, for $x \in \mathbb{R}^{n}$ and $a>0$ we define

$$
x^{\downarrow a}=\left(x_{1}, \ldots, x_{n-1}, x_{n}-a\right) .
$$


THEOREM 4.1. For $x, y \in S_{a, b}$ and $\mu \geqslant \frac{1}{2}$ we have

$$
\begin{aligned}
& G_{S_{a, b}}^{(\mu)}(x, y) \\
& \quad \stackrel{\mu, n}{\approx} \frac{x_{n}^{\mu-1 / 2}}{y_{n}^{\mu+3 / 2}} \frac{e^{-\frac{\pi}{b}|x-y|}}{|x-y|^{n}} \frac{\left[\delta_{b}\left(x_{1}\right) \delta_{b}\left(y_{1}\right)\right] \wedge|x-y|^{2}}{\left(\frac{1}{b}|x-y|+\cosh \rho_{a}\right)} \frac{\left(1+\frac{1}{b}|x-y|\right)^{n / 2+\mu+3 / 2}}{\left(\frac{1}{b}|x-y|+\cosh \rho\right)^{\mu-1 / 2}},
\end{aligned}
$$

where $\rho_{a}$ is the hyperbolic distance between $x^{\downarrow a}$ and $y^{\downarrow a}$.

Proof. By the scaling property, $G_{S_{a, b}}^{(\mu)}(x, y)=b^{-n} G_{S_{a / b, 1}}^{(\mu)}(x / b, y / b)$, hence it is enough to consider $b=1$. Furthermore, Theorem 3.3 (ii) gives

$$
G_{S_{a, 1}}^{(\mu)}(x, y)=\frac{1}{y_{n}^{2}} \int_{0}^{\infty} j\left(t ; x_{1}, y_{1}\right) \frac{\exp \left(-\frac{1}{2 t} \sum_{k=2}^{n-1}\left(x_{k}-y_{k}\right)^{2}\right)}{(2 \pi t)^{(n-2) / 2}} g_{a}^{(-\mu)}\left(t ; x_{n}, y_{n}\right) d t
$$

where $g_{a}^{(-\mu)}\left(t ; x_{n}, y_{n}\right)$ is the transition density of the Bessel process with index $-\mu$ killed on exiting $(a, \infty)$ and $j\left(t ; x_{1}, y_{1}\right)$ is the transition density function of a one-dimensional Brownian motion killed on exiting the interval $(0,1)$. Estimates of the function $j\left(t ; x_{1}, y_{1}\right)$ are given in [21, Theorem 5.4] (cf. [11, (5.7), p. 341] and [21, Theorem 2.2]):

$$
\begin{aligned}
& j\left(t ; x_{1}, y_{1}\right) \\
& \approx\left(1 \wedge \frac{x_{1} y_{y}}{t}\right)\left(1 \wedge \frac{\left(1-x_{1}\right)\left(1-y_{1}\right)}{t}\right) \frac{1+t^{5 / 2}}{\sqrt{t}} e^{-\pi^{2} t / 2-\left(x_{1}-y_{1}\right)^{2} /(2 t)} \\
& \approx \frac{x_{1} y_{1}}{t+x_{1} y_{1}} \frac{\left(1-x_{1}\right)\left(1-y_{1}\right)}{t+\left(1-x_{1}\right)\left(1-y_{1}\right)} \frac{1+t^{5 / 2}}{\sqrt{t}} e^{-\pi^{2} t / 2-\left(x_{1}-y_{1}\right)^{2} /(2 t)} .
\end{aligned}
$$

Combining (4.2) with (2.7), we obtain

$$
\begin{aligned}
G_{S_{a, 1}}^{(\mu)}(x, y) \approx & x_{1} y_{1}\left(1-x_{1}\right)\left(1-y_{1}\right)\left(x_{n}-a\right)\left(y_{n}-a\right) x_{n}^{2 \mu-1} y_{n}^{-2} \\
& \times \int_{0}^{\infty} \frac{1}{t+x_{1} y_{1}} \frac{1}{t+\left(1-x_{1}\right)\left(1-y_{1}\right)} \frac{1+t^{5 / 2}}{t^{n / 2}} \frac{e^{-\pi^{2} t / 2-|x-y|^{2} /(2 t)}}{t+\left(x_{n}-a\right)\left(y_{n}-a\right)} \\
& \times\left(\frac{1}{t+x_{n} y_{n}}\right)^{\mu-1 / 2} d t .
\end{aligned}
$$

Next, we apply Lemma 5.1 with $\alpha=5 / 2, \beta=(n-2) / 2, b=|x-y|^{2}, k=4$, $a_{1}=x_{1} y_{1}, \gamma_{1}=1, a_{2}=\left(1-x_{1}\right)\left(1-y_{1}\right), \gamma_{2}=1, a_{3}=\left(x_{n}-a\right)\left(y_{n}-a\right)$, $\gamma_{3}=1, a_{4}=x_{n} y_{n}, \gamma_{4}=\mu-1 / 2$ to get

$$
\begin{aligned}
& G_{S_{a, 1}}^{(\mu)}(x, y) \\
& \quad \stackrel{\mu, n}{\approx} \frac{x_{n}^{\mu-1 / 2}}{y_{n}^{\mu+3 / 2}} \frac{\delta_{1}\left(x_{1}\right) \delta_{1}\left(y_{1}\right)(1+|x-y|)^{\mu+n / 2+7 / 2} e^{-\pi|x-y|^{2}}}{|x-y|^{n-2}\left(|x-y|+\cosh \rho_{a}\right)(|x-y|+\cosh \rho)^{\mu-1 / 2}} w(x, y),
\end{aligned}
$$


where

$$
\begin{aligned}
w(x, y)= & \frac{1}{x_{1} y_{1}+x_{1} y_{1}|x-y|+|x-y|^{2}} \\
& \times \frac{1}{\left(1-x_{1}\right)\left(1-y_{1}\right)+\left(1-x_{1}\right)\left(1-y_{1}\right)|x-y|+|x-y|^{2}} .
\end{aligned}
$$

To complete the proof we need to show that

$$
w(x, y) \approx \frac{\left(\delta_{1}\left(x_{1}\right) \delta_{1}\left(y_{1}\right)\right) \wedge|x-y|^{2}}{\delta_{1}\left(x_{1}\right) \delta_{1}\left(y_{1}\right)|x-y|^{2}\left(1+|x-y|^{2}\right)} .
$$

For $\left|x_{1}-y_{1}\right| \geqslant 1 / 2$ we get $w(x, y) \approx 1 /|x-y|^{4}$, which is equivalent to 4.4 in this case. On the other hand, for $|x-y|<1 / 2$ we have $x_{1} y_{1} \approx 1$ or $\left(1-x_{1}\right)\left(1-y_{1}\right) \approx 1$ and consequently

$$
\begin{aligned}
w(x, y) & \approx \frac{1}{x_{1} y_{1}+|x-y|^{2}} \frac{1}{\left(1-x_{1}\right)\left(1-y_{1}\right)+|x-y|^{2}} \\
& \approx \frac{1}{\left[\left(1-x_{1}\right)\left(1-y_{1}\right)\right] \wedge\left[x_{1} y_{1}\right]+|x-y|^{2}} \approx \frac{1}{\delta_{1}\left(x_{1}\right) \delta_{1}\left(y_{1}\right)+|x-y|^{2}}
\end{aligned}
$$

as required.

The Poisson kernel of smooth and bounded domains may be obtained as the normal derivative of the Green function. Since the set $S_{a, b}$ is neither bounded nor smooth, we estimate its Poisson kernel separately.

THEOREM 4.2. For $x \in S_{a, b}, y \in \partial S_{a, b}$ and $\mu \geqslant 1 / 2$ we have

$$
\begin{aligned}
P_{S_{a, b}}^{(\mu)}(x, y) \stackrel{\mu, n}{\approx} & \left(\frac{x_{n}}{y_{n}}\right)^{\mu-1 / 2} \frac{e^{-\frac{\pi}{b}|x-y|}\left(1+\frac{1}{b}|x-y|\right)^{\mu+(n+3) / 2}}{|x-y|^{n}\left(\frac{1}{b}|x-y|+\cosh \rho\right)^{\mu-1 / 2}} \\
& \times \begin{cases}\frac{\delta_{b}\left(x_{1}\right)}{\frac{1}{b}|x-y|+\cosh \rho_{a}}, & y_{1} \in\{0, b\}, \\
\left(x_{n}-y_{n}\right) \frac{\left[\delta_{b}\left(x_{1}\right) \delta_{b}\left(y_{1}\right)\right] \wedge|x-y|^{2}}{|x-y|^{2}}, & y_{n}=a .\end{cases}
\end{aligned}
$$

Proof. In view of the scaling property and Theorem 3.3 we only need to investigate the density function of $Y\left(\tau_{S_{a, 1}}^{Y}\right)$. Recall that $Y=\left(B_{1}, \ldots, B_{n-1}, R^{(-\mu)}\right)$ is introduced in 2.12). Let $\tau_{(0,1)}^{B}$ be the first exit time from $(0,1)$ by the onedimensional Brownian motion $B_{1}(t)$ and $\tau_{(a, \infty)}^{R}$ be the first exit time from $(a, \infty)$ by the Bessel process $R^{(-\mu)}(t)$. Observe that

$$
\tau_{S_{a, 1}}^{Y}=\tau_{(0,1)}^{B} \wedge \tau_{(a, \infty)}^{R} .
$$


Furthermore, let us divide the boundary $\partial S_{a, 1}$ of $S_{a, 1}$ into two parts: $\partial_{1} S_{a, 1}=$ $\{0,1\} \times \mathbb{R}^{n-2} \times(a, \infty)$ and $\partial_{2} S_{a, 1}=(0,1) \times \mathbb{R}^{n-2} \times\{a\}$. For any Borel subset $A \subset \partial_{1} S_{a, 1}$ we have

$$
\begin{aligned}
& \mathbb{P}^{x}\left(Y\left(\tau_{S_{a, 1}}^{Y}\right) \in A\right) \\
& \quad=\mathbb{P}^{x}\left(\left(B_{1}\left(\tau_{(0,1)}^{B}\right), \ldots, B_{n-1}\left(\tau_{(0,1)}^{B}\right), R^{(-\mu)}\left(\tau_{(0,1)}^{B}\right)\right) \in A, \tau_{(0,1)}^{B} \leqslant \tau_{(a, \infty)}^{R}\right) .
\end{aligned}
$$

Since $\tau_{(0,1)}^{B}$ is independent of the rest of the above-appearing processes and variables, we may write

$$
\begin{aligned}
& \mathbb{P}^{x}\left(Y\left(\tau_{S_{a, 1}}^{Y}\right) \in A\right) \\
& \quad=\int_{A} \int_{0}^{\infty} \gamma\left(t ; x_{1}, y_{1}\right) \frac{\exp \left(-\frac{1}{2 t} \sum_{k=2}^{n-1}\left(x_{k}-y_{k}\right)^{2}\right)}{(2 \pi t)^{(n-2) / 2}} g_{a}^{(\mu)}\left(t ; x_{n}, y_{n}\right) d t d y,
\end{aligned}
$$

where $\gamma\left(t ; x_{1}, y_{1}\right)=\mathbb{P}^{x}\left(B_{1}\left(\tau_{(0,1)}^{B}\right)=y_{1}, \tau_{(0,1)}^{B} \in d t\right) / d t$. Consequently, the inner integral represents the Poisson kernel $P_{S_{a, 1}}^{(\mu)}(x, y)$. Using the estimates (see [21, Thm. 5.3])

$$
\gamma\left(t ; x_{1}, y_{1}\right) \approx x_{1}\left(1-x_{1}\right) \frac{1+t^{5 / 2}}{\left(t+1-\left|x_{1}-y_{1}\right|\right) t^{3 / 2}} \exp \left(-\frac{\left|x_{1}-y_{1}\right|^{2}}{2 t}-\frac{1}{2} \pi^{2} t\right),
$$

where $x_{1}, y_{1} \in(0,1), t>0$, and the formula 2.7$)$, we obtain

$$
\begin{aligned}
& P_{S_{a, 1}}^{(\mu)}(x, y) \stackrel{\mu, n}{\approx} x_{1}\left(1-x_{1}\right)\left(x_{n}-a\right)\left(y_{n}-a\right) x_{n}^{2 \mu-1} \\
& \quad \times \int_{0}^{\infty} \frac{1+t^{5 / 2}}{\left(t+1-\left|x_{1}-y_{1}\right|\right) t^{(n+2) / 2}} \frac{\exp \left(-\frac{|x-y|^{2}}{2 t}-\frac{1}{2} \pi^{2} t\right)}{t+\left(x_{n}-a\right)\left(y_{n}-a\right)}\left(\frac{1}{t+x_{n} y_{n}}\right)^{\mu-1 / 2} d t .
\end{aligned}
$$

To deal with this integral, we apply Lemma 5.1 with $\alpha=5 / 2, \beta=n / 2, b=$ $|x-y|^{2}, k=3, a_{1}=1-\left|x_{1}-y_{1}\right|, \gamma_{1}=1, a_{2}=\left(x_{n}-a\right)\left(y_{n}-a\right), \gamma_{2}=1$, $a_{3}=x_{n} y_{n}, \gamma_{3}=\mu-1 / 2$ to get

$$
\begin{aligned}
P_{S_{a, 1}}^{(\mu)}(x, y) \stackrel{\mu, n}{\approx} & \frac{x_{1}\left(1-x_{1}\right) e^{-\pi|x-y|}(1+|x-y|)^{\mu+(n+7) / 2}}{|x-y|^{n}\left(1+|x-y|+\frac{|x-y|^{2}}{\left(x_{n}-a\right)\left(y_{n}-a\right)}\right)\left(1+|x-y|+\frac{|x-y|^{2}}{x_{n} y_{n}}\right)^{\mu-1 / 2}} \\
& \times\left(\frac{x_{n}}{y_{n}}\right)^{\mu-1 / 2} \frac{1}{1-\left|x_{1}-y_{1}\right|+\left(1-\left|x_{1}-y_{1}\right|\right)|x-y|+|x-y|^{2}} .
\end{aligned}
$$

Using $1+|x-y|-\left|x_{1}-y_{1}\right| \approx 1+|x-y|$, we estimate the last denominator as follows:

$$
\begin{aligned}
1-\left|x_{1}-y_{1}\right|+ & \left(1-\left|x_{1}-y_{1}\right|\right)|x-y|+|x-y|^{2} \\
& =\left(1-\left|x_{1}-y_{1}\right|+|x-y|\right)-\left|x_{1}-y_{1}\right||x-y|+|x-y|^{2} \\
& \approx 1+|x-y|\left(1-\left|x_{1}-y_{1}\right|+|x-y|\right) \approx 1+|x-y|^{2} .
\end{aligned}
$$


Eventually we arrive at

$P_{S_{a, 1}}^{(\mu)}(x, y) \stackrel{\mu, n}{\approx}\left(\frac{x_{n}}{y_{n}}\right)^{\mu-1 / 2} \frac{\delta_{1}\left(x_{1}\right) e^{-\pi|x-y|}(1+|x-y|)^{\mu+(n+3) / 2}}{|x-y|^{n}\left(|x-y|+\cosh \rho_{a}\right)(|x-y|+\cosh \rho)^{\mu-1 / 2}}$,

which completes the estimates of the Poisson kernel on the first part of the boundary. Assume now $B \subset \partial_{2} S_{a, 1}$. Note that $y_{n}=a$ for $y \in \partial_{2} S_{a, 1}$. As in the previous case, we get

$$
\begin{aligned}
\mathbb{P}^{x}\left(Y\left(\tau_{S_{a, 1}}^{Y}\right) \in A\right) & =\mathbb{P}^{x}\left(\left(B_{1}\left(\tau_{(a, \infty)}^{R}\right), \ldots, B_{n-1}\left(\tau_{(a, \infty)}^{R}\right), a\right) \in A, \tau_{(a, \infty)}^{R}<\tau_{(0,1)}^{\beta}\right) \\
= & \int_{B} \int_{0}^{\infty} j\left(t ; x_{1}, y_{1}\right) \frac{\exp \left(-\frac{1}{2 t} \sum_{k=2}^{n-1}\left(x_{k}-y_{k}\right)^{2}\right)}{(2 \pi t)^{(n-2) / 2}} q_{a}^{(-\mu)}\left(t ; x_{n}\right) d t d y,
\end{aligned}
$$

where $q_{a}^{(-\mu)}\left(t ; x_{n}\right)=\mathbb{P}^{x_{n}}\left(\tau_{(a, \infty)}^{R} \in d t\right) / d t$. Hence, by 4.2 and 2.8 , we obtain

$$
\begin{aligned}
P_{S_{a, 1}}^{(\mu)}(x, y) \stackrel{\mu, n}{\approx} & x_{1} y_{1}\left(1-x_{1}\right)\left(1-y_{1}\right)\left(x_{n}-y_{n}\right) x_{n}^{2 \mu-1} \\
& \times \int_{0}^{\infty} \frac{1+t^{5 / 2}}{t+x_{1} y_{1}} \frac{e^{-\pi^{2} t / 2-|x-y|^{2} / 2 t}}{t+\left(1-x_{1}\right)\left(1-y_{1}\right)} \frac{1}{t^{(n+2) / 2}} \frac{1}{\left(t+y_{n} x_{n}\right)^{\mu-1 / 2}} d t .
\end{aligned}
$$

Next, we apply Lemma 5.1 with $\alpha=5 / 2, \beta=n / 2, b=|x-y|^{2}, k=3$, $a_{1}=x_{1} y_{1}, \gamma_{1}=1, a_{2}=\left(1-x_{1}\right)\left(1-y_{1}\right), \gamma_{2}=1, a_{3}=y_{n} x_{n}, \gamma_{3}=\mu-1 / 2$ to get

$$
\begin{aligned}
& P_{S_{a, 1}}^{(\mu)}(x, y) \\
& \stackrel{\mu, n}{\approx} \frac{x_{1} y_{1}\left(1-x_{1}\right)\left(1-y_{1}\right)\left(x_{n}-y_{n}\right) x^{2 \mu-1}}{\left(y_{n} x_{n}+y_{n} x_{n}|x-y|+|x-y|^{2}\right)^{\mu-1 / 2} e^{-\pi|x-y|}} \frac{(1+|x-y|)^{\mu+(n+7) / 2}}{|x-y|^{n}} w(x, y) \\
& \approx\left(\frac{x_{n}}{y_{n}}\right)^{\mu-1 / 2} \frac{\delta_{1}\left(x_{1}\right) \delta_{1}\left(y_{1}\right)\left(x_{n}-y_{n}\right) e^{-\pi|x-y|}}{|x-y|^{n}} \frac{(1+|x-y|)^{\mu+(n+7) / 2}}{(|x-y|+\cosh \rho)^{\mu-1 / 2}} w(x, y),
\end{aligned}
$$

where $w(x, y)$ is given by 4.3 . Applying 4.4 ends the proof.

Manipulating the parameters $a$ and $b$ in Theorems 4.1 and 4.2, we obtain some further results. Letting $a \rightarrow 0$ and using monotone convergence we get the corollary below. It generalizes estimates from [22], where HBM without drift was considered.

COROllary 4.1. For $x, y \in S_{0, b}$ we have

$$
\begin{aligned}
& G_{S_{0, b}}^{(\mu)}(x, y) \\
& \quad \stackrel{\mu, n}{\approx} \frac{x_{n}^{\mu-1 / 2}}{y_{n}^{\mu+3 / 2}} \frac{e^{-\frac{\pi}{b}|x-y|}}{|x-y|^{n}}\left(\delta_{b}\left(x_{1}\right) \delta_{b}\left(y_{1}\right) \wedge|x-y|^{2}\right) \frac{\left(1+\frac{1}{b}|x-y|\right)^{n / 2+\mu+3 / 2}}{\left(\frac{1}{b}|x-y|+\cosh \rho\right)^{\mu+1 / 2}},
\end{aligned}
$$


and for $x \in S_{0, b}$ and $y \in \partial S_{0, b}$ we have

$$
\begin{aligned}
& P_{S_{0, b}}^{(\mu)}(x, y) \\
& \stackrel{\mu, n}{\approx}\left\{\begin{array}{l}
\left(\frac{x_{n}}{y_{n}}\right)^{\mu-1 / 2} \frac{\delta_{b}\left(x_{1}\right) e^{-\frac{\pi}{b}|x-y|}\left(1+\frac{1}{b}|x-y|\right)^{\mu+(n+3) / 2}}{|x-y|^{n}\left(\frac{1}{b}|x-y|+\cosh \rho\right)^{\mu+1 / 2}}, \quad y_{1} \in\{0, b\}, \\
x_{n}^{2 \mu} e^{-\frac{\pi}{b}|x-y|}\left(\delta_{b}\left(x_{1}\right) \delta_{b}\left(y_{1}\right) \wedge|x-y|^{2}\right) \frac{\left(1+\frac{1}{b}|x-y|\right)^{\mu+(n+3) / 2}}{|x-y|^{2 \mu+n+1}}, \quad y_{n}=a .
\end{array}\right.
\end{aligned}
$$

Letting then $b \rightarrow \infty$ we obtain estimates provided in [17]. The next corollary concerns the most studied subset of $\mathbb{H}^{n}$ in the context of HBM, i.e. $D_{a}=$ $\left\{x \in \mathbb{H}^{n}: x_{n}>a\right\}, a>0$. It follows from Theorems 4.1 and 4.2 by replacing $x_{1}$ and $y_{1}$ by $x_{1}+b / 2$ and $y_{1}+b / 2$, respectively, and letting $b \rightarrow \infty$. In fact, the Poisson kernel $P_{D_{a}}^{(\mu)}(x, y)$ was estimated in [8], and estimates of the $\lambda$-Green function for the process without drift (which, by Theorem 3.1, are equivalent to estimates of the Green function for the process with a suitable drift) are the main results of [3].

COROllary 4.2. For $\mu>0$ we have

$$
\begin{aligned}
& G_{D_{a}}^{(\mu)}(x, y) \stackrel{\mu, n}{\approx} \frac{x_{n}^{\mu-1 / 2}}{y_{n}^{\mu+3 / 2}} \frac{1}{|x-y|^{n-2} \cosh \rho_{a}(\cosh \rho)^{\mu-1 / 2}}, \quad x, y \in D_{a}, \\
& P_{D_{a}}^{(\mu)}(x, y) \stackrel{\mu, n}{\approx}\left(\frac{x_{n}}{y_{n}}\right)^{\mu-1 / 2} \frac{x_{n}-y_{n}}{|x-y|^{n}(\cosh \rho)^{\mu-1 / 2}}, \quad x \in D_{a}, y \in \partial D_{a} .
\end{aligned}
$$

\section{APPENDIX}

In this section we present a technical lemma which is used to estimate integrals appearing in Section 4.

LEMMA 5.1. For fixed $\alpha \geqslant 0, \beta \geqslant 1 / 2, k \in \mathbb{N}, \gamma_{i} \geqslant 0$ for $i \in\{1, \ldots, k-1\}$ and $\gamma_{k}>-1 / 2$ define

$$
J\left(a_{1}, \ldots, a_{k}, b\right)=\int_{0}^{\infty} \frac{(1+t)^{\alpha}}{t^{\beta+1}} \frac{\exp \left(-\frac{b^{2}}{2 t}-\frac{1}{2} \pi^{2} t\right)}{\prod_{i=1}^{k}\left(a_{i}+t\right)^{\gamma_{i}}} d t, \quad a_{1}, \ldots, a_{k}, b>0
$$

There exists a constant $c=c\left(\alpha, \beta, \gamma_{1}, \ldots, \gamma_{k}\right)$ such that

$$
J\left(a_{1}, \ldots, a_{k}, b\right) \approx \frac{c}{b^{2 \beta}} \frac{(1+b)^{\alpha+\beta-1 / 2+\sum_{i=1}^{k} \gamma_{i}}}{\prod_{i=1}^{k}\left(a_{i}+a_{i} b+b^{2}\right)^{\gamma_{i}}} .
$$


Proof. Throughout this proof, $c$ represents a constant depending on $\alpha, \beta$, $\gamma_{1}, \ldots, \gamma_{k}$. Substituting $t=b u / \pi$ in the integral from the statement we get

$$
\frac{e^{-b \pi}}{b^{\beta}} \pi^{\beta-\alpha+\sum_{i=1}^{k} \gamma_{i}} \int_{0}^{\infty} \frac{(\pi+u b)^{\alpha}}{u^{\beta+1}} \frac{\exp \left(-\frac{1}{2} b \pi\left(\sqrt{u}-\frac{1}{\sqrt{u}}\right)^{2}\right)}{\prod_{i=1}^{k}\left(a_{i} \pi+b u\right)^{\gamma_{i}}} d u=: \frac{e^{-b \pi}}{b^{\beta}} \mathcal{I} .
$$

It is enough to show that

$$
\mathcal{I} \stackrel{c}{\approx} \frac{(1+b)^{\alpha+\beta-1 / 2+\sum_{i=1}^{k} \gamma_{i}}}{b^{\beta} \prod_{i=1}^{k}\left(a_{i}+a_{i} b+b^{2}\right)^{\gamma_{i}}} .
$$

We start with the substitution $\sqrt{u}-\frac{1}{\sqrt{u}}=s \sqrt{\frac{2}{b}}$. Note that

$$
u=\left(\sqrt{1+\frac{s^{2}}{2 b}}+\frac{s}{\sqrt{2 b}}\right)^{2} \approx \begin{cases}1+s^{2} / b, & s>0(\Leftrightarrow u>1), \\ \frac{1}{1+s^{2} / b}, & s \leqslant 0(\Leftrightarrow u \leqslant 1),\end{cases}
$$

and

$$
\frac{d u}{u}=\frac{2 d s}{\sqrt{s^{2}+2 b}} \approx \frac{d s}{\sqrt{s^{2}+b}} .
$$

Consequently,

$$
\begin{aligned}
\mathcal{I} \stackrel{c}{\approx} b^{\beta} \int_{0}^{\infty} \frac{\left(1+b+s^{2}\right)^{\alpha}}{\left(b+s^{2}\right)^{\beta+1 / 2}} \frac{e^{-s^{2}}}{\prod_{i=1}^{k}\left(a_{i}+b+s^{2}\right)^{\gamma_{i}}} d s \\
\quad+\frac{1}{b^{\beta}} \int_{-\infty}^{0} \frac{\left(s^{2}+b\right)^{\beta-1 / 2}\left(1+\frac{b^{2}}{s^{2}+b}\right)^{\alpha} e^{-s^{2}}}{\prod_{i=1}^{k}\left(a_{i}+\frac{b^{2}}{s^{2}+b}\right)^{\gamma_{i}}} d s \\
=: b^{\beta} \mathcal{I}_{1}+\frac{1}{b^{\beta}} \mathcal{I}_{2} .
\end{aligned}
$$

For $b>1$ we have

$$
\mathcal{I}_{1}=\frac{(1+b)^{\alpha}}{b^{\beta+1 / 2} \prod_{i=1}^{k}\left(a_{i}+b\right)^{\gamma_{i}}} \int_{0}^{\infty} \frac{\left(1+\frac{s^{2}}{1+b}\right)^{\alpha}}{\left(1+\frac{s^{2}}{b}\right)^{\beta+1 / 2}} \frac{e^{-s^{2}}}{\prod_{i=1}^{k}\left(1+\frac{s^{2}}{a_{i}+b}\right)^{\gamma_{i}}} d s .
$$

Since $\alpha \geqslant 0$ and $\beta \geqslant 1 / 2$, the integral above may be bounded from above by

$$
\int_{0}^{\infty} \frac{\left(1+s^{2}\right)^{\alpha} e^{-s^{2}}}{\prod_{i=1}^{k}\left(1+s^{2} \mathbf{1}_{(-\infty, 0)}\left(\gamma_{i}\right)\right)^{\gamma_{i}}} d s,
$$

and from below by

$$
\int_{0}^{\infty} \frac{1}{\left(1+s^{2}\right)^{\beta+1 / 2}} \frac{e^{-s^{2}}}{\prod_{i=1}^{k}\left(1+s^{2} \mathbf{1}_{(0, \infty)}\left(\gamma_{i}\right)\right)^{\gamma_{i}}} d s .
$$


Hence, estimating $b+1 \approx b$, we get

$$
\mathcal{I}_{1} \stackrel{c}{\approx} \frac{b^{\alpha-\beta-1 / 2}}{\prod_{i=1}^{k}\left(a_{i}+b\right)^{\gamma_{i}}}
$$

Furthermore, using $0 \leqslant s^{2} / b \leqslant s^{2}$ and $0 \leqslant a_{i} s^{2} /\left[b\left(a_{i}+b\right)\right]<s^{2}$, we have

$$
\begin{aligned}
\mathcal{I}_{2} & =\int_{0}^{\infty} \frac{\left(b\left(\frac{s^{2}}{b}+1\right)\right)^{\beta-1 / 2}\left(1+b \frac{b}{s^{2}+b}\right)^{\alpha} e^{-s^{2}}}{\prod_{i=1}^{k}\left(a_{i}+b\right)^{\gamma_{i}}} \prod_{i=1}^{k}\left(\frac{1+s^{2} / b}{1+\frac{a_{i} s^{2}}{b\left(a_{i}+b\right)}}\right)^{\gamma_{i}} d s \\
& \leqslant \frac{b^{\beta-1 / 2}(1+b)^{\alpha}}{\prod_{i=1}^{k}\left(a_{i}+b\right)^{\gamma_{i}}} \int_{0}^{\infty}\left(s^{2}+1\right)^{\beta-1 / 2+\sum_{i=1}^{k}\left|\gamma_{i}\right|} e^{-s^{2}} d s \stackrel{c}{\approx} \frac{b^{\alpha+\beta-1 / 2}}{\prod_{i=1}^{k}\left(a_{i}+b\right)^{\gamma_{i}}}
\end{aligned}
$$

Hence

$$
\mathcal{I} \stackrel{c}{\approx} b^{\beta} \mathcal{I}_{1} \stackrel{c}{\approx} \frac{b^{\alpha-1 / 2}}{\prod_{i=1}^{k}\left(a_{i}+b\right)^{\gamma_{i}}}, \quad b>1
$$

which is equivalent to 5.1. Assume now $b \leqslant 1$. We have

$$
\mathcal{I}_{2} \stackrel{c}{\approx} \int_{-\infty}^{0} \frac{\left(s^{2}+b\right)^{\beta-1 / 2} e^{-s^{2}}}{\prod_{i=1}^{k}\left(a_{i}+\frac{b^{2}}{s^{2}+b}\right)^{\gamma_{i}}} d s
$$

We are going to use the inequalities $s^{2}<s^{2}+b<s^{2}+1$ and

$$
\left(a_{i}+b^{2}\right) \frac{1}{1+s^{2}}<a_{i}+\frac{b^{2}}{s^{2}+b}<\left(a_{i}+b^{2}\right)\left(1+\frac{1}{s^{2}}\right), \quad 1 \leqslant i \leqslant k .
$$

Note that, as long as all $\gamma_{i}$ are nonnegative, replacing $a_{i}+\frac{b^{2}}{s^{2}+b}$ by $\left(a_{i}+b^{2}\right)\left(1+\frac{1}{s^{2}}\right)$ does not affect integrability of the above integral. However, it works also if $\gamma_{k} \in$ $(-1 / 2,0)$. Thus

$$
\mathcal{I}_{2} \stackrel{c}{\approx} \frac{1}{\prod_{i=1}^{k}\left(a_{i}+b^{2}\right)^{\gamma_{i}}}, \quad b \leqslant 1
$$

It is now enough to show that $b^{\beta} \mathcal{I}_{1} \leqslant c b^{-\beta} \mathcal{I}_{2}$ for $b \leqslant 1$. We rewrite $\mathcal{I}_{1}$ as follows:

$$
\begin{aligned}
\mathcal{I}_{1} & =\left(\int_{0}^{1}+\int_{1}^{\infty}\right) \frac{\left(1+b+s^{2}\right)^{\alpha}}{\left(b+s^{2}\right)^{\beta+1 / 2}} \frac{e^{-s^{2}}}{\prod_{i=1}^{k}\left(a_{i}+b+s^{2}\right)^{\gamma_{i}}} d s \\
& \approx \int_{0}^{1} \frac{1}{\left(b+s^{2}\right)^{\beta+1 / 2}} \frac{d s}{\prod_{i=1}^{k}\left(a_{i}+b+s^{2}\right)^{\gamma_{i}}}+\int_{1}^{\infty} \frac{s^{2 \alpha-2 \beta-1} e^{-s^{2}}}{\prod_{i=1}^{k}\left(a_{i}+s^{2}\right)^{\gamma_{i}}} d s \\
& =: \mathcal{I}_{1,1}+\mathcal{I}_{1,2} .
\end{aligned}
$$


In order to estimate $\mathcal{I}_{1,1}$ we substitute $s=\sqrt{b} u$ to get

$$
\begin{aligned}
\mathcal{I}_{1,1} & =\frac{b^{-\beta / 2-1 / 4}}{\prod_{i=1}^{k}\left(a_{i}+b\right)^{\gamma_{i}}} \int_{0}^{1 / \sqrt{b}} \frac{1}{\left(1+u^{2}\right)^{\beta+1 / 2}} \frac{d u}{\prod_{i=1}^{k}\left(1+\frac{b}{a_{i}+b} u^{2}\right)^{\gamma_{i}}} \\
& \leqslant \frac{b^{-\beta / 2-1 / 4}}{\prod_{i=1}^{k}\left(a_{i}+b\right)^{\gamma_{i}}} \int_{0}^{\infty} \frac{1}{\left(1+u^{2}\right)^{\beta+1 / 2}} \frac{d u}{\left(1+\frac{b}{a_{i_{0}}+b} u^{2}\right)^{\gamma_{k}}} \\
& \leqslant \frac{b^{-\beta / 2-1 / 4}}{\prod_{i=1}^{k}\left(a_{i}+b\right)^{\gamma_{i}}} \int_{0}^{\infty} \frac{d u}{\left(1+u^{2}\right)^{\beta+1 / 2+\mathbf{1}_{(-\infty, 0)}\left(\gamma_{k}\right)}},
\end{aligned}
$$

where the last integral is convergent by the assumptions $\beta>1 / 2$ and $\gamma_{k}>-1 / 2$. The same bound may be obtained for $\mathcal{I}_{1,2}$. Indeed, due to the inequalities $a_{i}+1 \leqslant$ $a_{i}+s^{2} \leqslant\left(a_{i}+1\right)\left(1+s^{2}\right)$ for $s \geqslant 1$ we have

$$
\begin{aligned}
\mathcal{I}_{1,2} & \stackrel{c}{\approx} \frac{1}{\prod_{i=1}^{k}\left(a_{i}+1\right)^{\gamma_{i}}}=\frac{\prod_{i=1}^{k}\left(\frac{a_{i}+b}{a_{i}+1}\right)^{\gamma_{i}}}{\prod_{i=1}^{k}\left(a_{i}+b\right)^{\gamma_{i}}} \\
& \leqslant \frac{\left(\frac{a_{k}+1}{a_{k}+b}\right)^{1 / 2}}{\prod_{i=1}^{k}\left(a_{i}+b\right)^{\gamma_{i}}} \leqslant \frac{b^{-1 / 2}}{\prod_{i=1}^{k}\left(a_{i}+b\right)^{\gamma_{i}}} \leqslant \frac{b^{-\beta / 2-1 / 4}}{\prod_{i=1}^{k}\left(a_{i}+b\right)^{\gamma_{i}}}
\end{aligned}
$$

where we have used $\beta>1 / 2$ and $b \leqslant 1$. Combining $(5.3)$ and 5.4 gives us

$$
\mathcal{I}_{1} \leqslant c \frac{b^{-\beta / 2-1 / 4}}{\prod_{i=1}^{k}\left(a_{i}+b\right)^{\gamma_{i}}}, \quad 0<b \leqslant 1, a_{1}, \ldots, a_{k}>0 .
$$

Thus, since $3 \beta / 2-1 / 4>1 / 2$,

$$
b^{\beta} \mathcal{I}_{1} \leqslant c \frac{1}{b^{\beta}} \mathcal{I}_{2} \frac{b^{3 \beta / 2-1 / 4}}{\prod_{i=1}^{k}\left(\frac{a_{i}+b}{a_{i}+b^{2}}\right)^{\gamma_{i}}} \leqslant c \frac{1}{b^{\beta}} \mathcal{I}_{2} b^{1 / 2}\left(\frac{a_{k}+b}{a_{k}+b^{2}}\right)^{-\min \left\{0, \gamma_{k}\right\}} .
$$

For $\gamma_{k} \geqslant 0$ we bound $b^{1 / 2} \leqslant 1$ and for $\gamma_{k} \in(-1 / 2,0)$,

$$
b^{1 / 2}\left(\frac{a_{k}+b}{a_{k}+b^{2}}\right)^{-\gamma_{k}} \leqslant\left(\frac{b\left(a_{k}+b\right)}{a_{k}+b^{2}}\right)^{-\gamma_{k}} \leqslant 1
$$

which gives $b^{\beta} \mathcal{I}_{1} \leqslant c b^{-\beta} \mathcal{I}_{2}$, as required. Hence

$$
\mathcal{I} \stackrel{c}{\approx} \frac{1}{b^{\beta}} \mathcal{I}_{2} \stackrel{c}{\approx} \frac{1}{b^{\beta} \prod_{i=1}^{k}\left(a_{i}+b^{2}\right)^{\gamma_{i}}}, \quad 0<b \leqslant 1, a_{1}, \ldots, a_{k}>0 .
$$

This coincides with 5.1 and the proof is complete. 


\section{REFERENCES}

[1] P. Baldi, E. Casadio Tarabusi, and A. Figà-Talamanca, Stable laws arising from hitting distributions of processes on homogeneous trees and the hyperbolic half-plane, Pacific J. Math. 197 (2001), 257-273.

[2] P. Baldi, E. Casadio Tarabusi, A. Figà-Talamanca, and M. Yor, Non-symmetric hitting distributions on the hyperbolic half-plane and subordinated perpetuities, Rev. Mat. Iberoamer. 17 (2001), 587-605.

[3] K. Bogus, T. Byczkowski, and J. Małecki, Sharp estimates of the Green function of hyperbolic Brownian motion, Studia Math. 228 (2015), 197-221.

[4] K. Bogus and J. Małecki, Sharp estimates of transition probability density for Bessel process in half-line, Potential Anal. 43 (2015), 1-22.

[5] A. N. Borodin and P. Salminen, Handbook of Brownian Motion-Facts and Formulae, 2nd ed., Birkhäuser, Basel, 2002.

[6] T. Byczkowski, P. Graczyk, and A. Stós, Poisson kernels of half-spaces in real hyperbolic spaces, Rev. Mat. Iberoamer. 23 (2007), 85-126.

[7] T. Byczkowski and J. Małecki, Poisson kernel and Green function of the ball in real hyperbolic spaces, Potential Anal. 27 (2007), 1-26.

[8] T. Byczkowski, J. Małecki, and M. Ryznar, Hitting times of Bessel processes, Potential Anal. 38 (2013), 753-786.

[9] T. Byczkowski, J. Małecki, and T. Żak, Feynman-Kac formula, $\lambda$-Poisson kernels and $\lambda$-Green functions of half-spaces and balls in hyperbolic spaces, Colloq. Math. 118 (2010), 201-222.

[10] D. Dufresne, The distribution of a perpetuity, with application to risk theory and pension funding, Scand. Actuar. J. 25 (1990), 39-79.

[11] W. Feller, An Introduction to Probability Theory and its Applications. Vol. II, Wiley, New York, 1971.

[12] G. B. Folland, Fourier Analysis and its Applications, Wadsworth and Brooks, Pacific Grove, CA, 1992.

[13] C. Grosche and F. Steiner, Handbook of Feynman Path Integrals, Springer, Berlin, 1998.

[14] J.-C. Gruet, Semi-groupe du mouvement Brownien hyperbolique, Stoch. Stoch. Reports 56 (1996), 53-61.

[15] P. Hartman and G. S. Watson, "Normal" distribution functions on spheres and the modified Bessel functions, Ann. Probab. 2 (1974), 593-607.

[16] I. Karatzas and S. E. Shreve, Brownian Motion and Stochastic Calculus, Springer, New York, 1988.

[17] J. Małecki and G. Serafin, Hitting hyperbolic half-space, Demonstratio Math. 45 (2012), 337 360.

[18] H. Matsumoto, Closed formulae for the heat kernels and the Green functions for the Laplacians on the symmetric spaces of rank one, Bull. Sci. Math. 125 (2001), 553-581.

[19] S. A. Molchanov, On Martin boundaries for invariant Markov processes on a solvable group, Teor. Veroyatnost. i Primenen. 12 (1967), 358-362 (in Russian)

[20] S. A. Molchanov and E. Ostrovskii, Symmetric stable processes as traces of degenerate diffusion processes, Theory Probab. Appl. 12 (1969), 128-131.

[21] A. Pyć, G. Serafin, and T. Żak, Supremum distribution of Bessel process of drifting Brownian motion, Probab. Math. Statist. 35 (2015), 201-222.

[22] G. Serafin, Potential theory of hyperbolic Brownian motion in tube domains, Colloq. Math. 135 (2014), 27-52.

[23] B. Trojan, Asymptotic expansions and Hua-harmonic functions on bounded homogeneous domains, Math. Ann. 336 (2006), 73-110.

[24] M. Yor, Loi de l'indice du lacet Brownien, et distribution de Hartman-Watson, Z. Wahrsch. Verw. Gebiete 53 (1980), 71-95. 
[25] M. Yor, On some exponential functionals of Brownian motion, Adv. Appl. Probab. 24 (1992), $509-531$.

[26] M. Yor, Some Aspects of Brownian Motion, Part I: Some Special Functionals, Birkhäuser, Basel, 1992.

\section{Grzegorz Serafin}

Faculty of Pure and Applied Mathematics

Wrocław University of Science and Technology

Wybrzeże Wyspiańskiego 27

50-370 Wrocław, Poland

E-mail: grzegorz.serafin@pwr.edu.pl

Received 12.7.2017;

revised version 22.7.2018 